Müller-Gugenberger

Wirtschaftsstrafrecht 



\section{Wirtschafts- strafrecht}

Handbuch des Wirtschaftsstrafund -ordnungswidrigkeitenrechts

herausgegeben von

Dr. Christian Müller-Gugenberger

Richter am Oberlandesgericht a.D.

unter Mitwirkung von

Jens Gruhl

Leitender Oberstaatsanwalt

und

Anke Hadamitzky

Bundesanwältin beim Bundesgerichtshof

7. neu bearbeitete

und erweiterte Auflage

2021

\section{ottoschmidt}




\section{Zitierempfehlung:}

Bearbeiter in Müller-Gugenberger, Wirtschaftsstrafrecht, 7. Aufl., Rz. ...

\section{Bibliografische Information}

der Deutschen Nationalbibliothek

Die Deutsche Nationalbibliothek verzeichnet diese Publikation in der Deutschen Nationalbibliografie; detaillierte bibliografische Daten sind im Internet über http://dnb.d-nb.de abrufbar.

Verlag Dr. Otto Schmidt KG

Gustav-Heinemann-Ufer 58, 50968 Köln

Tel. 02 21/9 37 38-01, Fax 02 21/9 37 38-943

info@otto-schmidt.de

www.otto-schmidt.de

ISBN 978-3-504-40101-6

@ 2021 by Verlag Dr. Otto Schmidt KG, Köln

Das Werk einschließlich aller seiner Teile ist urheberrechtlich geschützt. Jede Verwertung, die nicht ausdrücklich vom Urheberrechtsgesetz zugelassen ist, bedarf der vorherigen Zustimmung des Verlages. Das gilt insbesondere für Vervielfältigungen, Bearbeitungen, Übersetzungen, Mikroverfilmungen und die Einspeicherung und Verarbeitung in elektronischen Systemen.

Das verwendete Papier ist aus chlorfrei gebleichten Rohstoffen hergestellt, holz- und säurefrei, alterungsbeständig und umweltfreundlich.

Einbandgestaltung: Lichtenford, Mettmann

Satz: WMTP, Birkenau

Druck und Verarbeitung: Kösel, Krugzell

Printed in Germany 


\section{Bearbeiter der 7. Auflage}

Dr. Thorsten Alexander

Rechtsanwalt, Stuttgart

Aniello Ambrosio

Staatsanwalt, Stuttgart

Manfred Büttner

Oberamtsrat, Lehrbeauftragter an der

Hochschule für Finanzen, Stuttgart

Dr. Joachim Dittrich

Leitender Oberstaatsanwalt, Stuttgart

Dr. Marc Engelhart

Rechtsanwalt, Referatsleiter am

Max-Planck-Institut für ausländisches und internationales Strafrecht, Freiburg i.Br.

Dr. Johannes Fridrich

Oberbürgermeister, Nürtingen,

Richter am Landgericht Stuttgart a.D.

\section{Gabriele Grätsch}

Staatsanwältin beim Bundesgerichtshof,

Karlsruhe

Dr. Andreas Grube

Richter am Bundesgerichtshof, Karlsruhe

Jens Gruhl

Leitender Oberstaatsanwalt, Hechingen

Dr. Markus Haas

Richter am Amtsgericht (sV),

Kirchheim unter Teck

Anke Hadamitzky

Bundesanwältin beim Bundesgerichtshof,

Referatsleiterin der Revisionsabteilung

beim Generalbundesanwalt, Karlsruhe

Klaus Heitmann

Richter am Landgericht a.D., Stuttgart

Oliver Henzler

Oberstaatsanwalt, Stuttgart

Peter Holzwarth

Oberstaatsanwalt, Stuttgart

Ilka Ludwig

Richterin am Landgericht, Besigheim
Prof. Dr. Manfred Muhler

Präsident des Finanzgerichts

Baden-Württemberg, Stuttgart

Dr. Christian Müller-Gugenberger

Richter am Oberlandesgericht a.D., Stuttgart

Dr. Alexander Nogrady

Oberstaatsanwalt, Leiter des Referats Internationale Zusammenarbeit in Strafsachen, Ministerium der Justiz und für Europa Baden-Württemberg, Stuttgart

Prof. Dr. Michael Pfohl

Leitender Oberstaatsanwalt a.D.,

Gomaringen, Honorarprofessor an der Juristischen Fakultät der Universität Tübingen

Dr. Alexander Retemeyer

Oberstaatsanwalt, Ständiger Vertreter des

Behördenleiters, Osnabrück

Dr. Hans Richter

Oberstaatsanwalt, Leiter der Abteilung

Wirtschaftsstrafsachen, a.D., Stuttgart

Wolfgang Schmid

Oberstaatsanwalt, Leiter der Abteilung

Wirtschaftsstrafsachen, a.D., Stuttgart

Andreas Thul

Oberstaatsanwalt, Stuttgart

Dr. Thomas Trück

Oberstaatsanwalt, Tübingen

Heiko Wagenpfeil

Oberstaatsanwalt, Stuttgart

Dr. Michael Wahl

Oberstaatsanwalt, Stuttgart

Dr. Beate Weik

Leitende Oberstaatsanwältin,

Generalstaatsanwaltschaft, Stuttgart

Prof. Dr. Wolfgang Winkelbauer

Rechtsanwalt, Stuttgart

Thomas Wolf

Stadtoberverwaltungsrat, Sindelfingen 


\section{Frühere Mitautoren}

Dr. Peter Bender

Finanzpräsident a.D., Rechtsanwalt,

Hannover

3. Aufl. 2000 bis 5. Aufl. 2011:

Zollrecht; Marktordnung; Subventionen

Klaus Bieneck † 2011

Oberstaatsanwalt a.D., Rechtsanwalt, Stuttgart 1. Aufl. 1987 bis 5. Aufl. 2011:

Konkurs bzw. Insolvenz; Auslandsgeschäfte; Kriegswaffen

3. Aufl. 2000 bis 5. Aufl. 2011:

Mitherausgeber

\section{Sandra Bischoff}

Leitende Oberstaatsanwältin, Stuttgart

5. Aufl. 2011 bis 6. Aufl. 2015:

Rechtshilfe

\section{Gernot Blessing}

Oberstaatsanwalt, Stuttgart

3. Aufl. 2000 bis 5. Aufl. 2011:

Arbeitnehmerschutz und Betriebsverfassung;

Korruption; Notstand

Dr. Johannes Häcker

Leitender Oberstaatsanwalt, Stuttgart

1. Aufl. 1987 bis 6. Aufl. 2015 :

Beteiligung; Berater

Ulrich Hebenstreit

Richter am Bundesgerichtshof, Karlsruhe 5. Aufl. 2011 bis 6. Aufl. 2015:

Betrug; Kreditbetrug;

Betrügerische Verkaufsmethoden

\section{Dr. Erwin Küster}

Regierungsdirektor, Finanzamt Stuttgart

1. Aufl. 1987 bis 3. Aufl. 2000:

Steuern einschließlich Verfahren

\section{Armin Nack}

Vorsitzender Richter am BGH

1. Aufl. 1987 bis 4. Aufl. 2006:

Betrug; Bargeldloser Zahlungsverkehr;

Kreditgewährung; Preisgestaltung;

Maßnahmen gegen Schuldner; Bank- und Börsengeschäfte; Organisierte Kriminalität u.a.
H.H. Jürgen Niemeyer

Vizepräsident des Landgerichts, Leipzig

1. Auff. 1987 bis 3. Aufl. 2000:

Geheimnisverletzungen; Korruption;

Unlautere Werbung

Dr. Jürgen Niemeyer

Vorsitzender Richter

am Oberlandesgericht, Stuttgart

3. Auff. 2000 bis 6. Aufl. 2015:

Verfahren; Strafrecht Allgemeiner Teil

einschließlich Sanktionen

Georg Prasser † 2007

Rechtsanwalt, Stuttgart

4. Aufl. 2006:

Verteidigung

Dr. Werner Schmidt-Hieber † 2011

Oberbürgermeister, Waiblingen

1. Aufl. 1987 bis 2. Aufl. 1992:

Sanktionen und Verfahren in

Wirtschaftssachen; Subventionen

3. Aufl. 2000:

Amtsuntreue

Dr. Alexander Schumann

Richter am Landgericht, Stuttgart

5. Aufl. 2011 bis 6. Aufl. 2015:

Bank und Börse

Ihre Beiträge zu den Vorauflagen wirken auch ohne Namensnennung - in der Neubearbeitung weiter. Darüber hinaus haben sie als Mitglieder unserer Autorengemeinschaft nachhaltig auf die Gestaltung und Strukturierung dieses Gemeinschaftswerks Einfluss genommen. (Titel und Berufsbezeichnungen sind auf dem Stand der letzten Auflage, an der sie jeweils mitgewirkt haben.) 